\title{
Extraction of Bionanomaterials from Aqueous Bulk by using Surface Active and Water Soluble Magnetic Nanoparticles
}

Rajpreet Kaur ${ }^{2,3}$, Poonam Khullar ${ }^{2}$, Anita Gupta ${ }^{3 *}$, Mandeep Singh Bakshi $^{1 *}$

${ }^{1}$ Department of Chemistry, Natural and Applied Sciences, University of Wisconsin - Green Bay, 2420 Nicolet Drive, Green Bay, WI 54311-7001, USA. ${ }^{2}$ Department of Chemistry, B.B.K. D.A.V. College for Women, Amritsar 143005, Punjab, India. ${ }^{3}$ Amity Institute of Applied Sciences, AUUP, Noida 201304, India. 
Fig S1

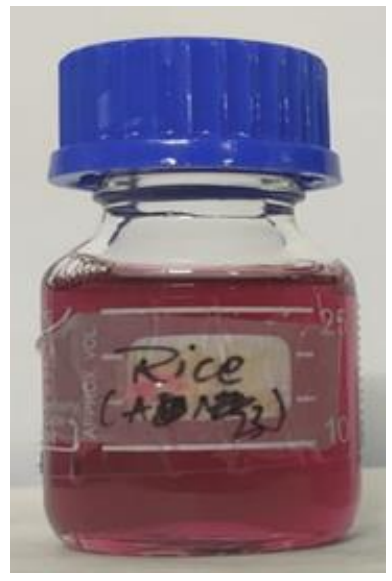

1

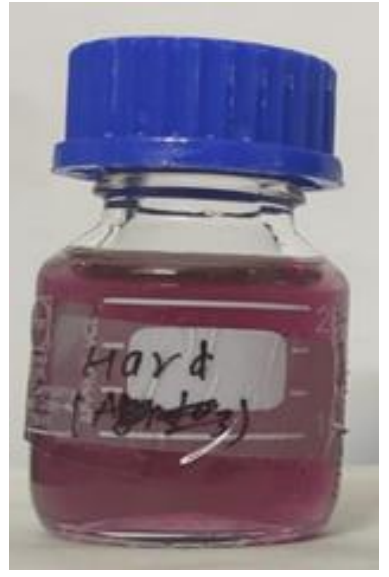

2

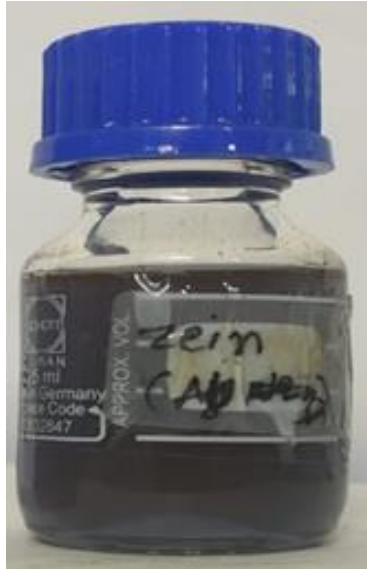

3

Fig S1. Rice (1), wheat (2), and zein (3) protein conjugated Au NPs. 
Fig S2

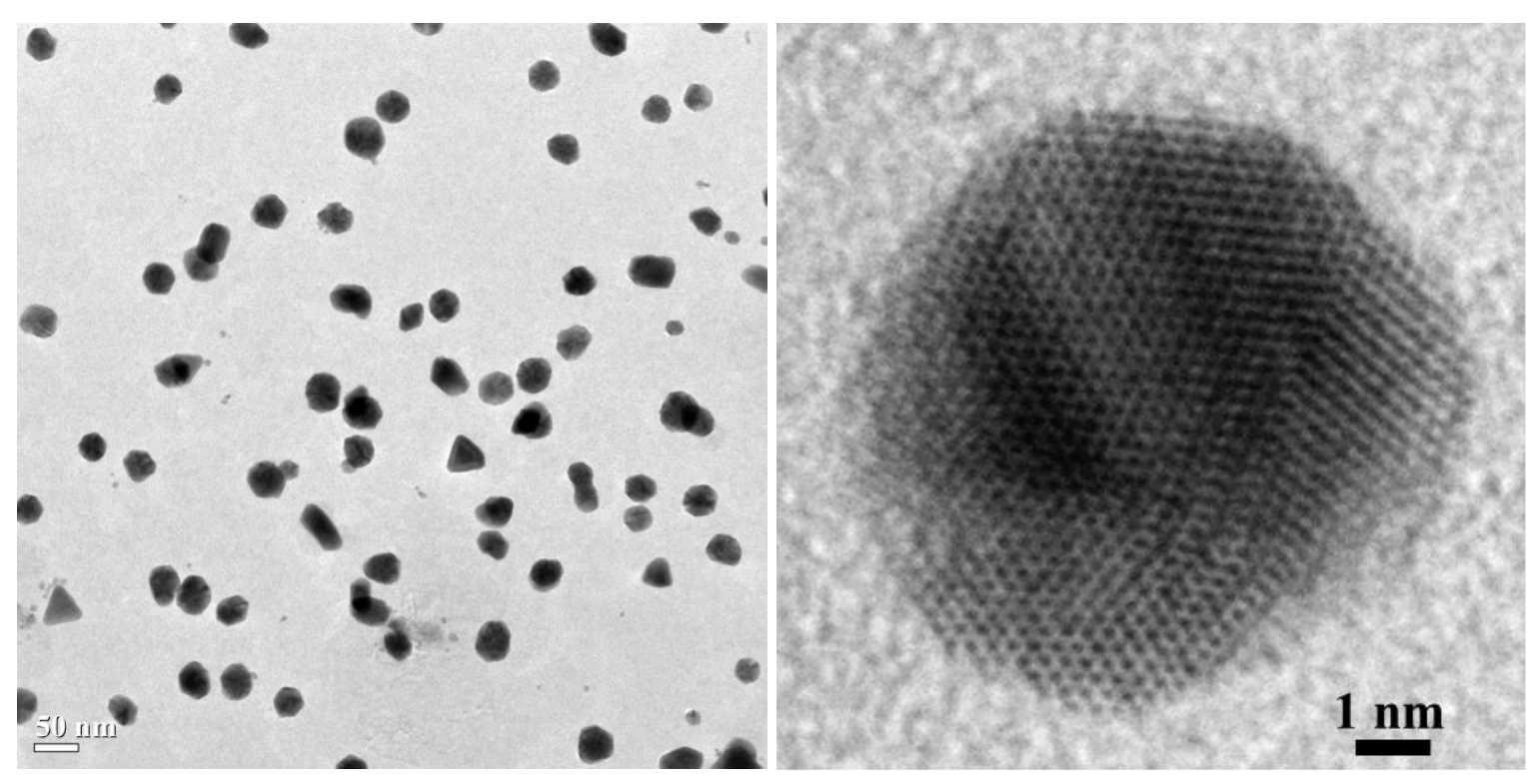

Fig S2. TEM image of rice protein conjugated Au NPs in low and high resolutions. 


\section{Fig S3}

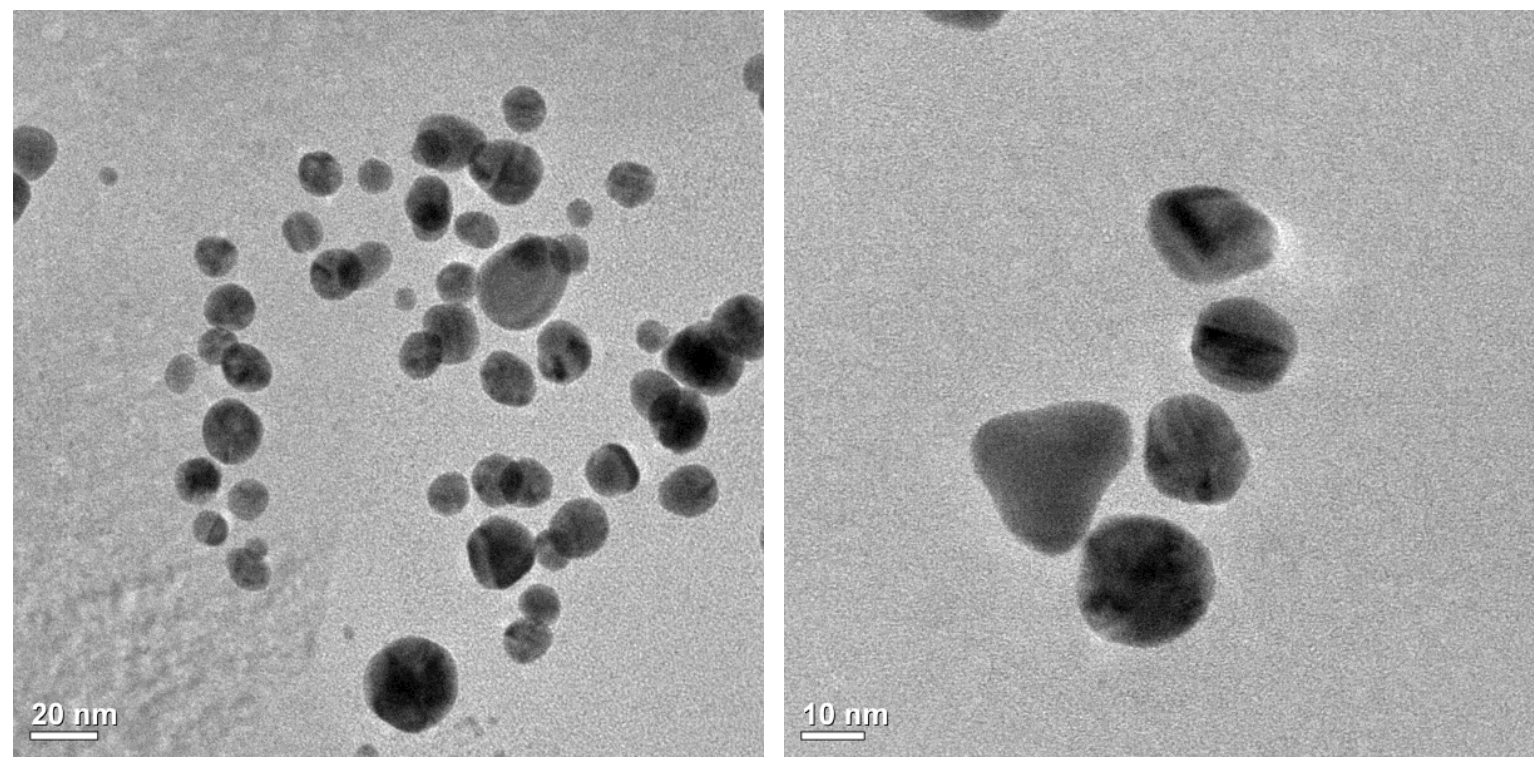

Fig S3. TEM image of wheat protein conjugated Au NPs in low and high resolutions. 
Fig S4

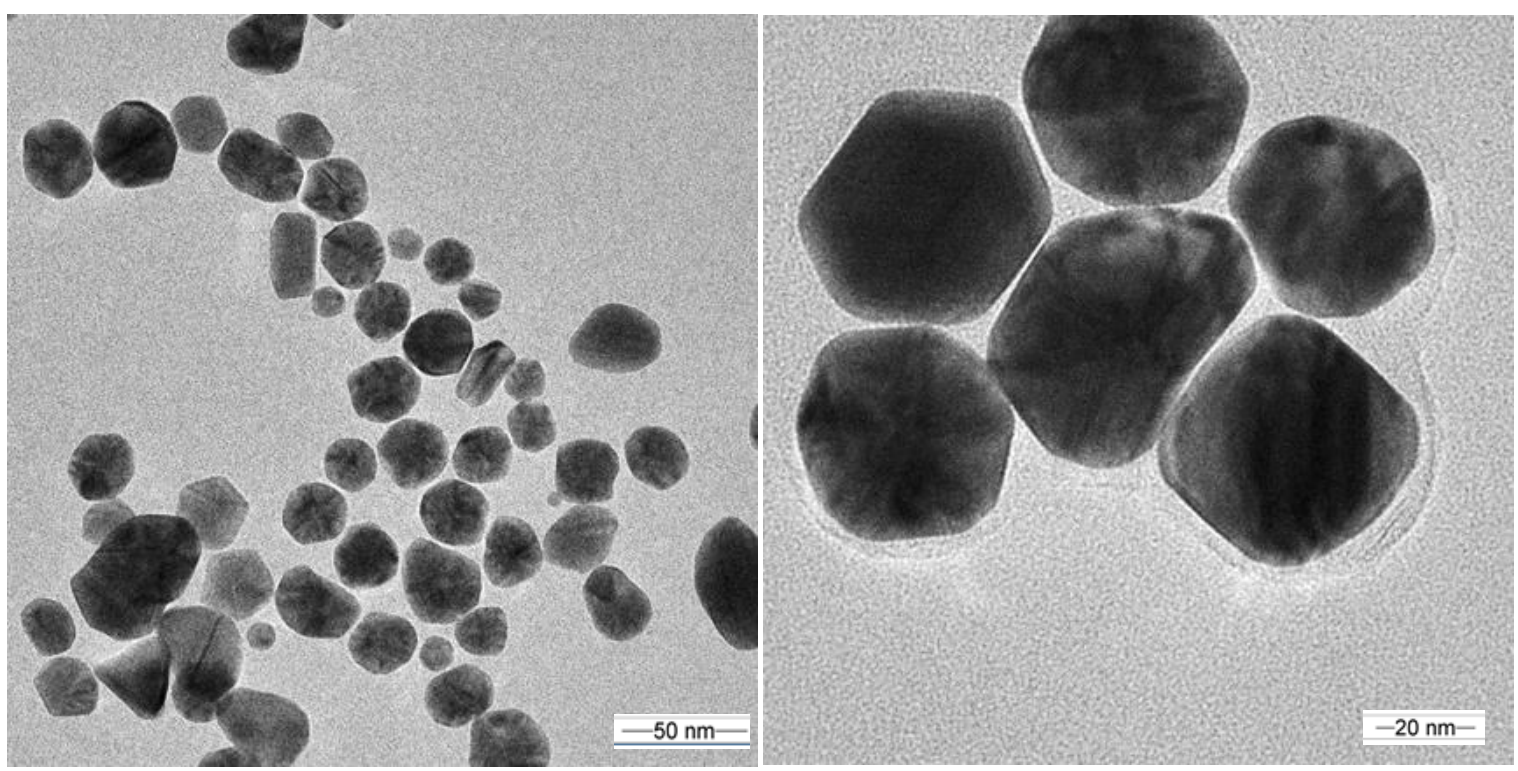

Fig S4. TEM image of zein protein conjugated Au NPs in low and high resolutions. 
Fig S5

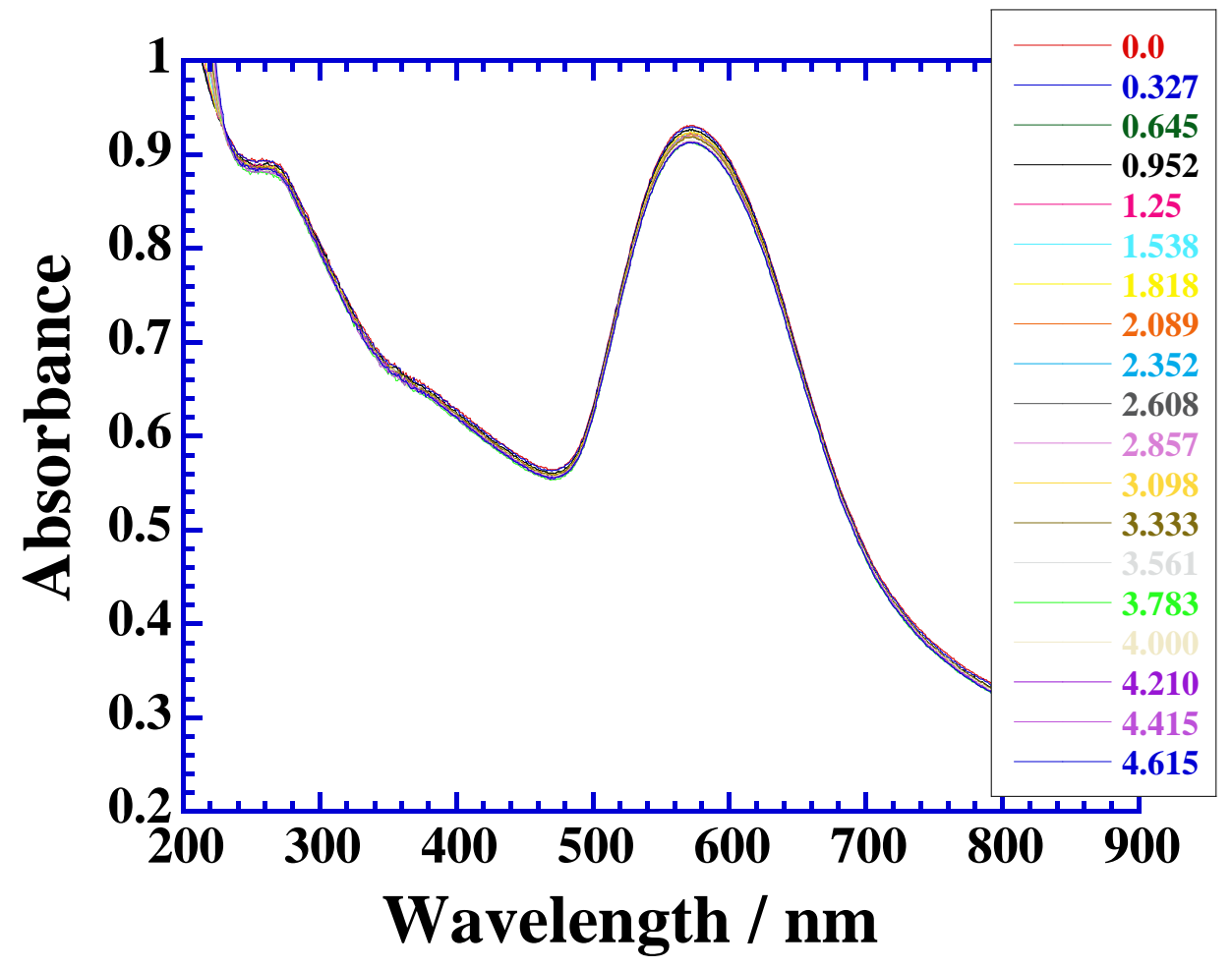

Fig S5. UV-visible scans with concentration/mM representing the extraction of zein protein conjugated Au NPs by placing SDS stabilized surface active magnetic NPs at aqueous - air interface. 
Fig S6

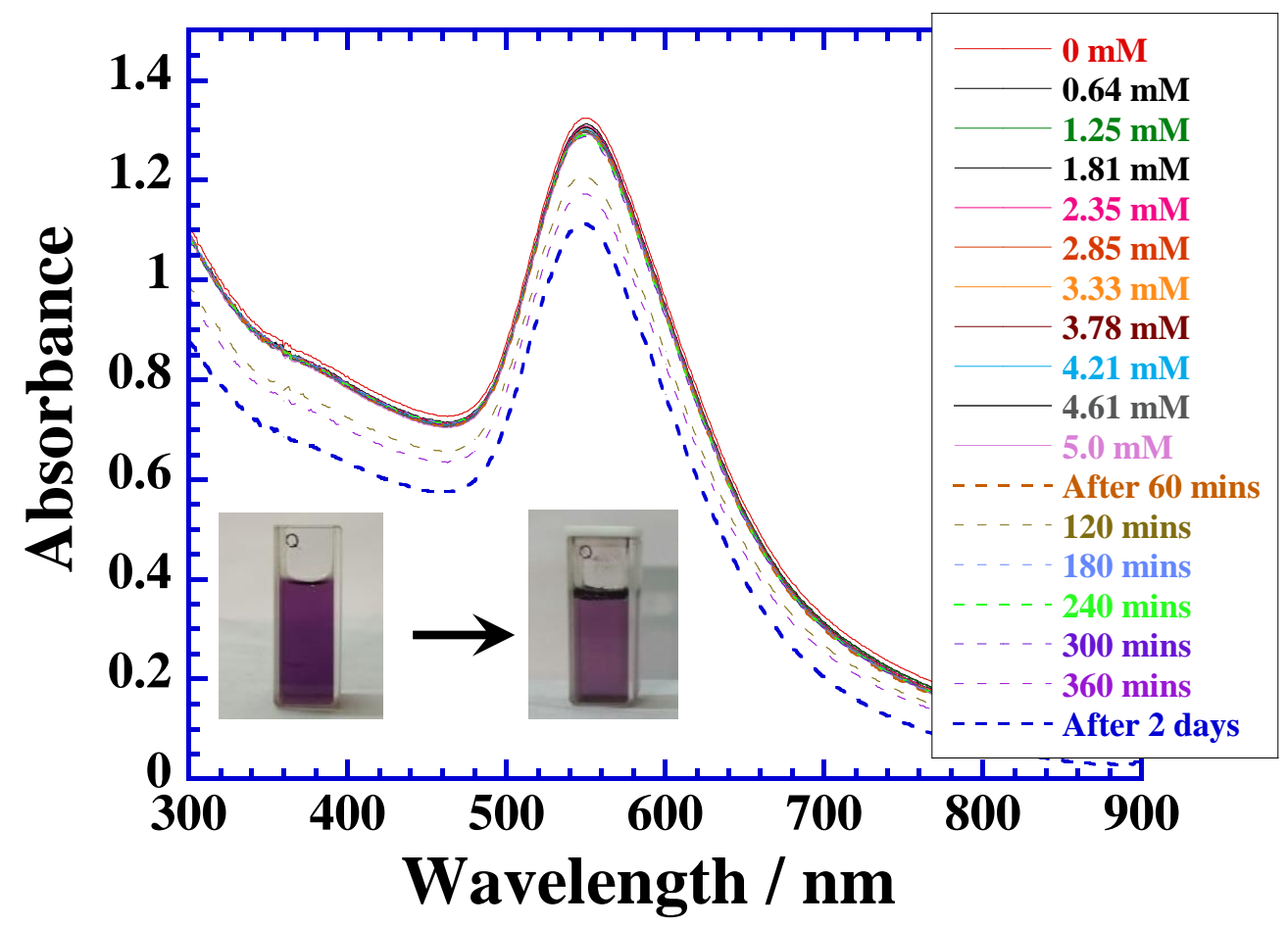

Fig S6. UV-visible scans with concentration/mM and time in minutes representing the extraction of zein protein conjugated Au NPs by placing 12-2-12 stabilized surface active magnetic NPs at aqueous - air interface. 

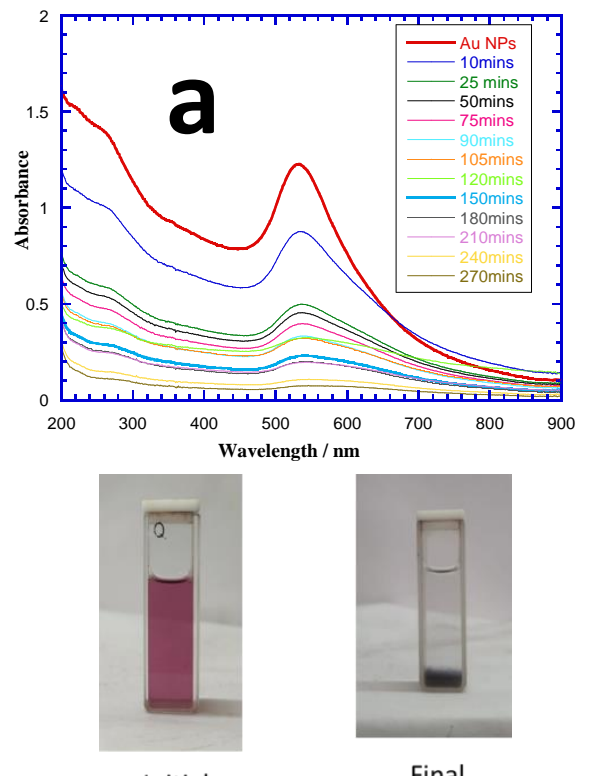

Initial

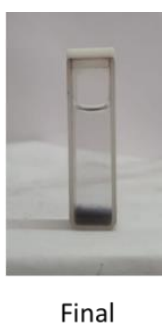

Final
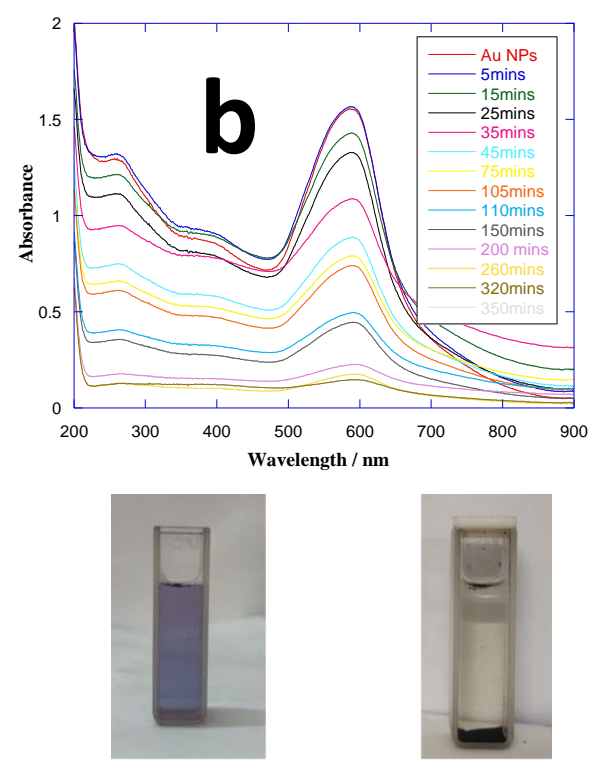

Initial

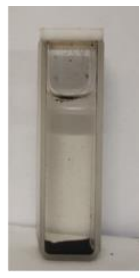

Final

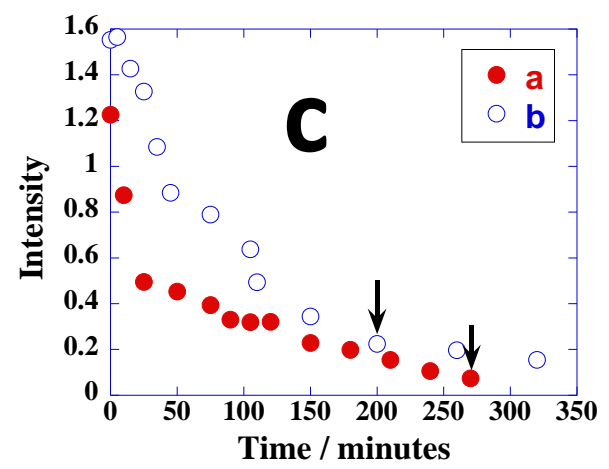

Fig S7. UV-visible scans with time in minutes representing the extraction of rice protein (in aqueous SDS, $\mathbf{a}$; in aqueous CTAB, $\mathbf{b}$ ) conjugated Au NPs by water soluble DPS stabilized magnetic NPs. (c) shows plots of intensity versus time of $\mathbf{a}$ and $\mathbf{b}$. Extraction is faster in (b) and is achieved within 200 minutes. 

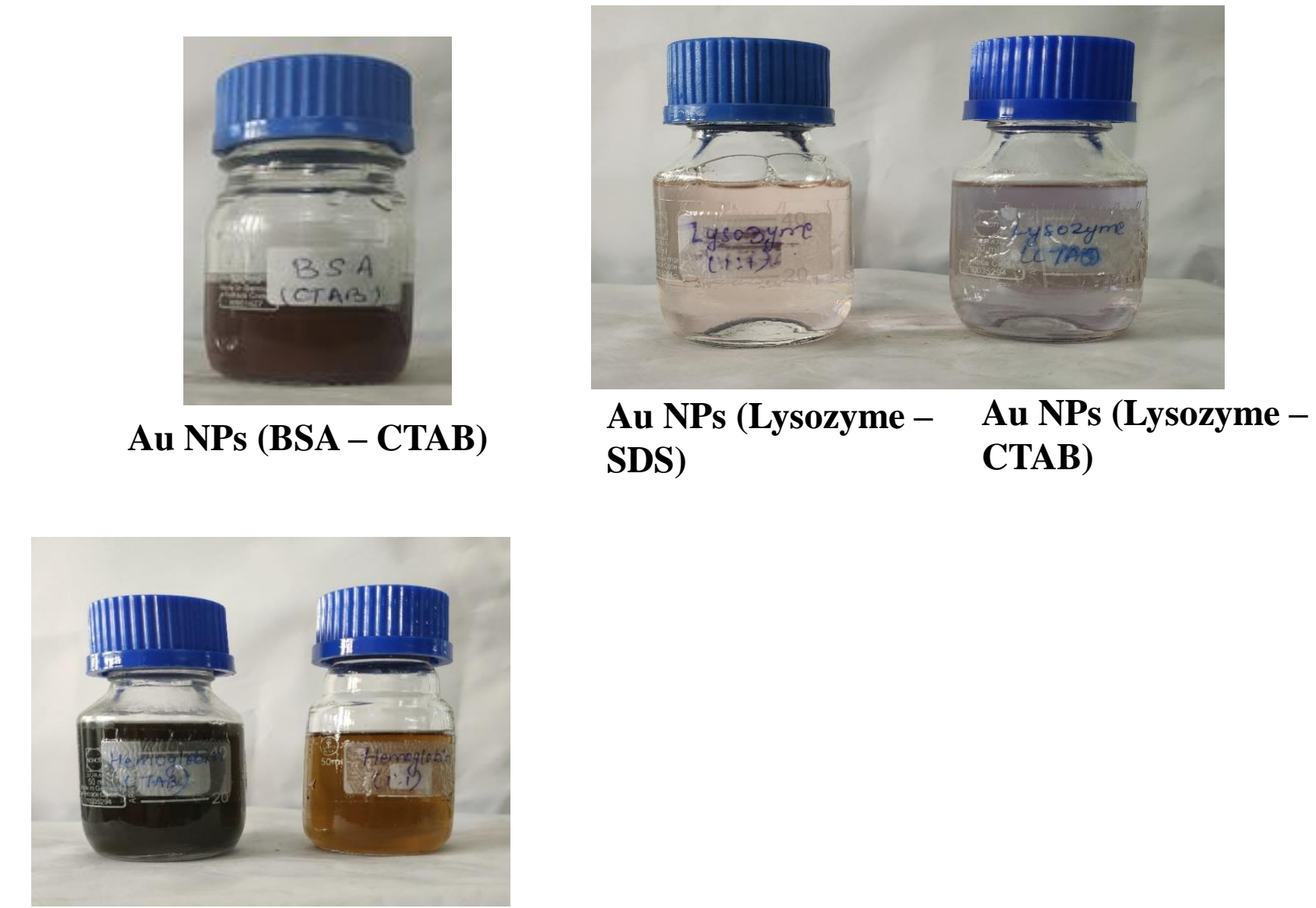

Au NPs (Hemoglobin - Au NPs (Hemoglobin CTAB) SDS)

Fig S8. BSA, lysozyme, and hemoglobin conjugated Au NPs. 

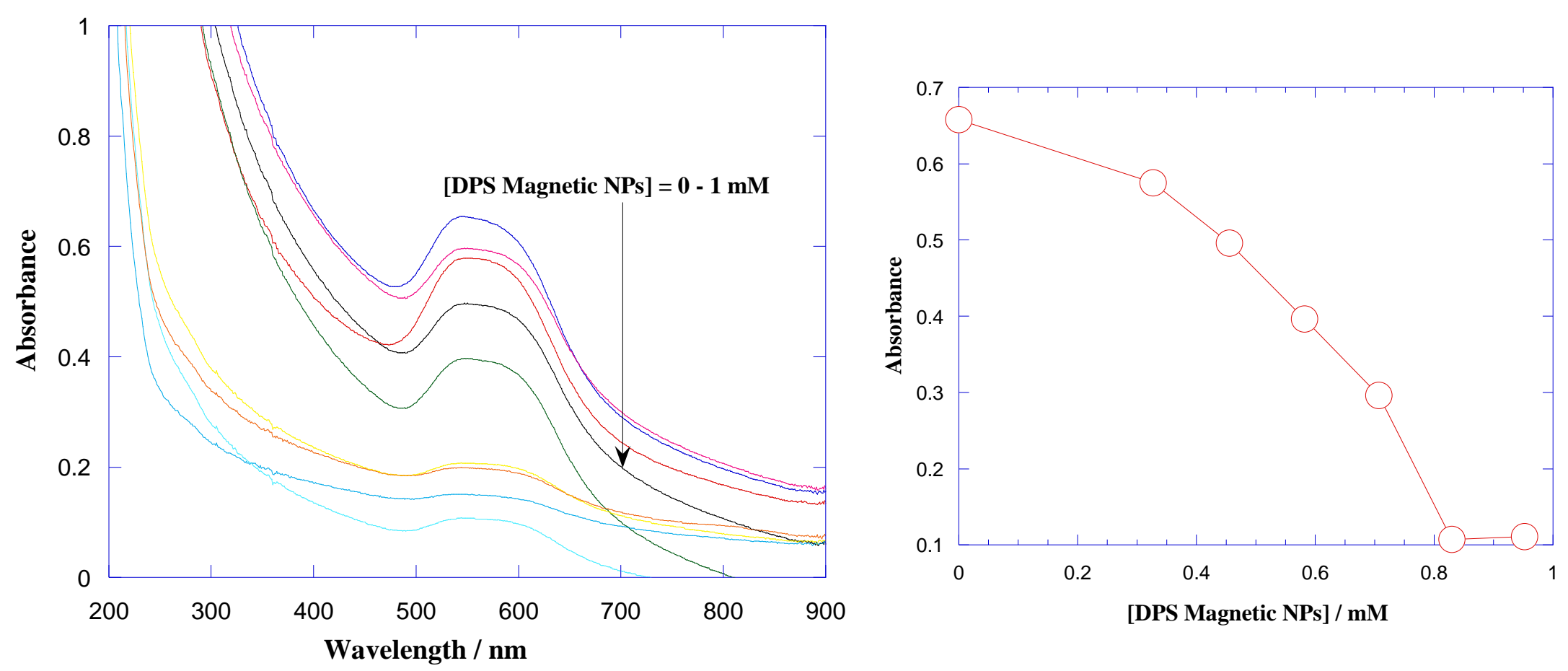

Fig S9. UV-visible scans (left frame) and intensity vs concentration / mM (right frame) representing the extraction of BSA (in pure water) conjugated Au NPs in the presence of DPS stabilized water soluble magnetic NPs in aqueous bulk. 

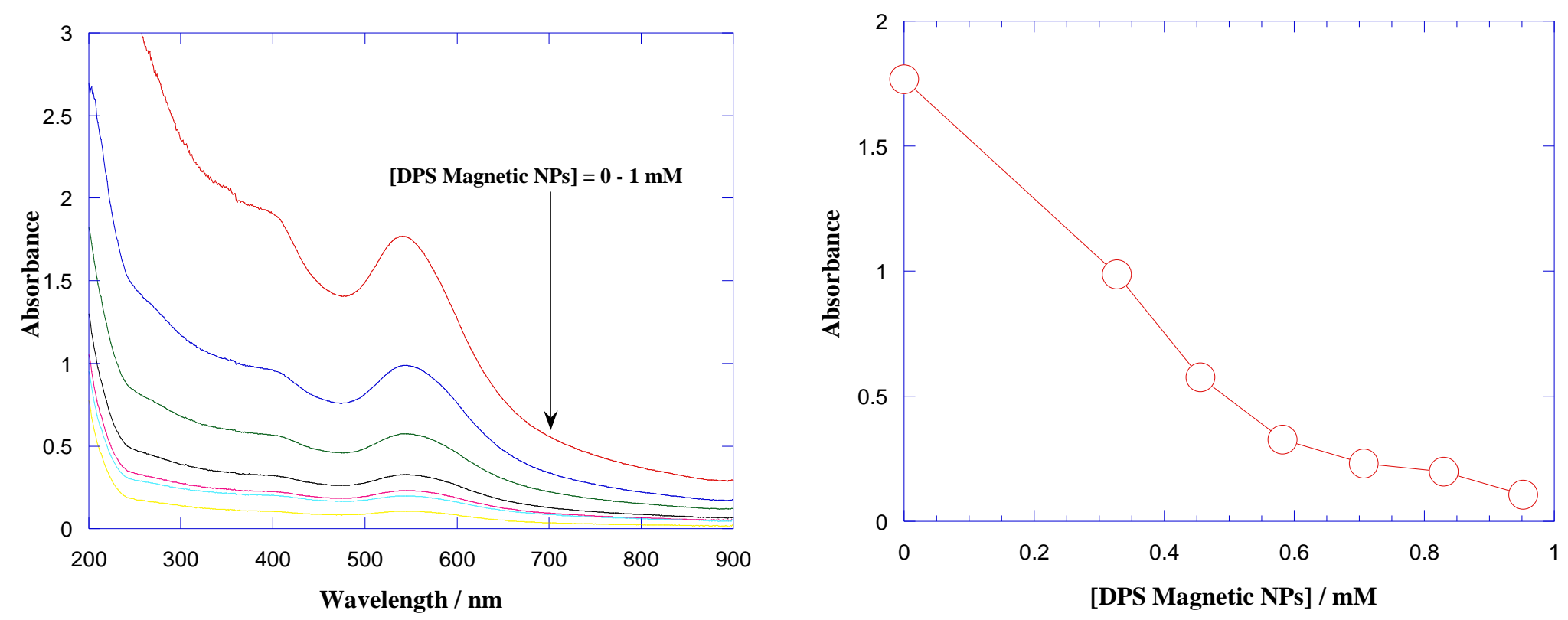

Fig S10. UV-visible scans (left frame) and intensity vs concentration / mM (right frame) representing the extraction of hemoglobin (in pure water) conjugated Au NPs in the presence of DPS stabilized water soluble magnetic NPs in aqueous bulk. 

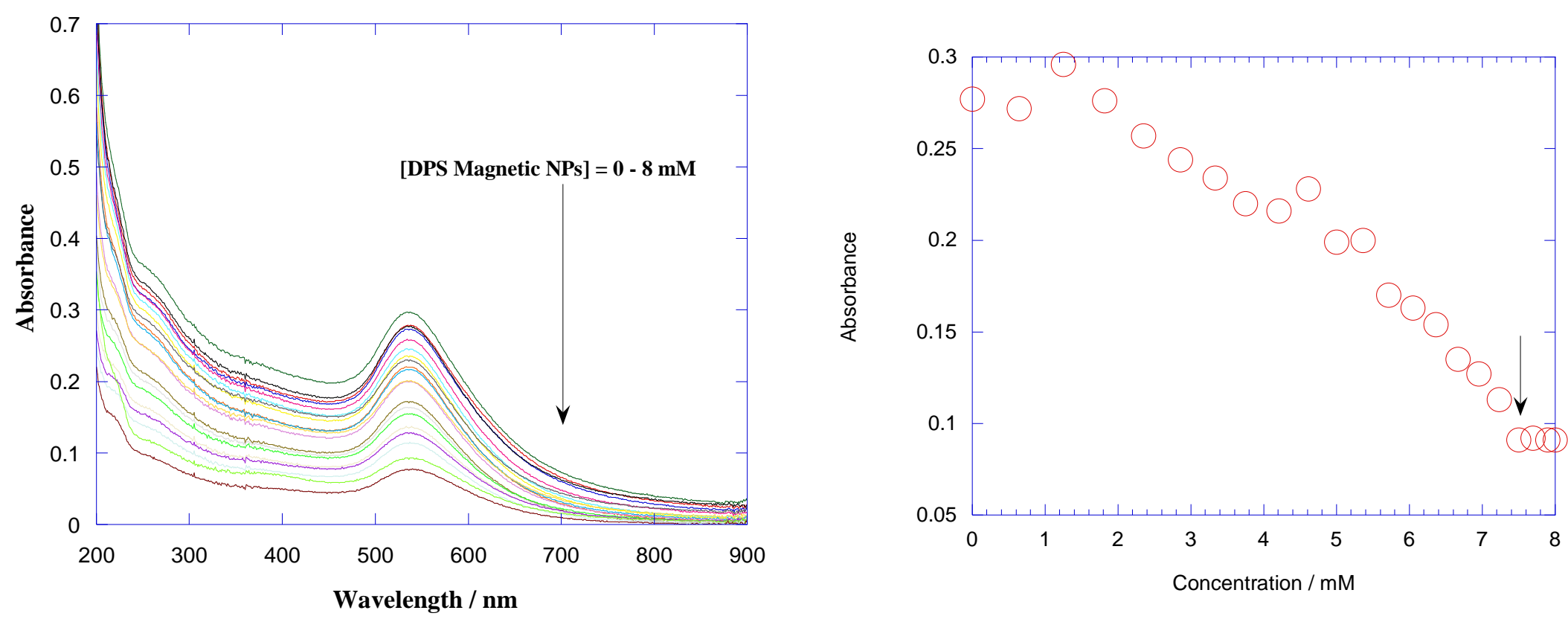

Fig S11. UV-visible scans (left frame) and intensity vs concentration / $\mathrm{mM}$ (right frame) representing the extraction of BSA (in aq SDS) conjugated Au NPs in the presence of DPS stabilized water soluble magnetic NPs in aqueous bulk. 


\section{Fig S12}
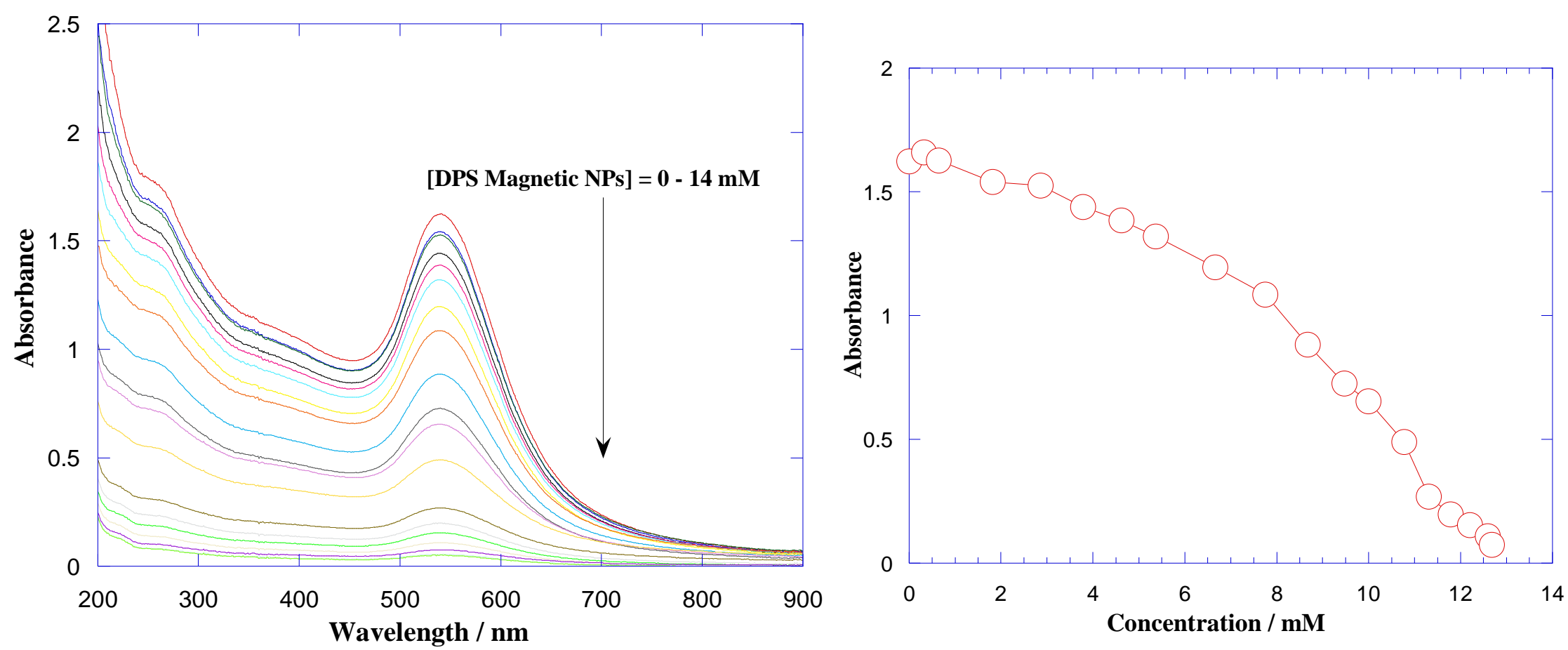

Fig S12. UV-visible scans (left frame) and intensity vs concentration / $\mathrm{mM}$ (right frame) representing the extraction of hemoglobin (in aq SDS) conjugated Au NPs in the presence of DPS stabilized water soluble magnetic NPs in aqueous bulk. 

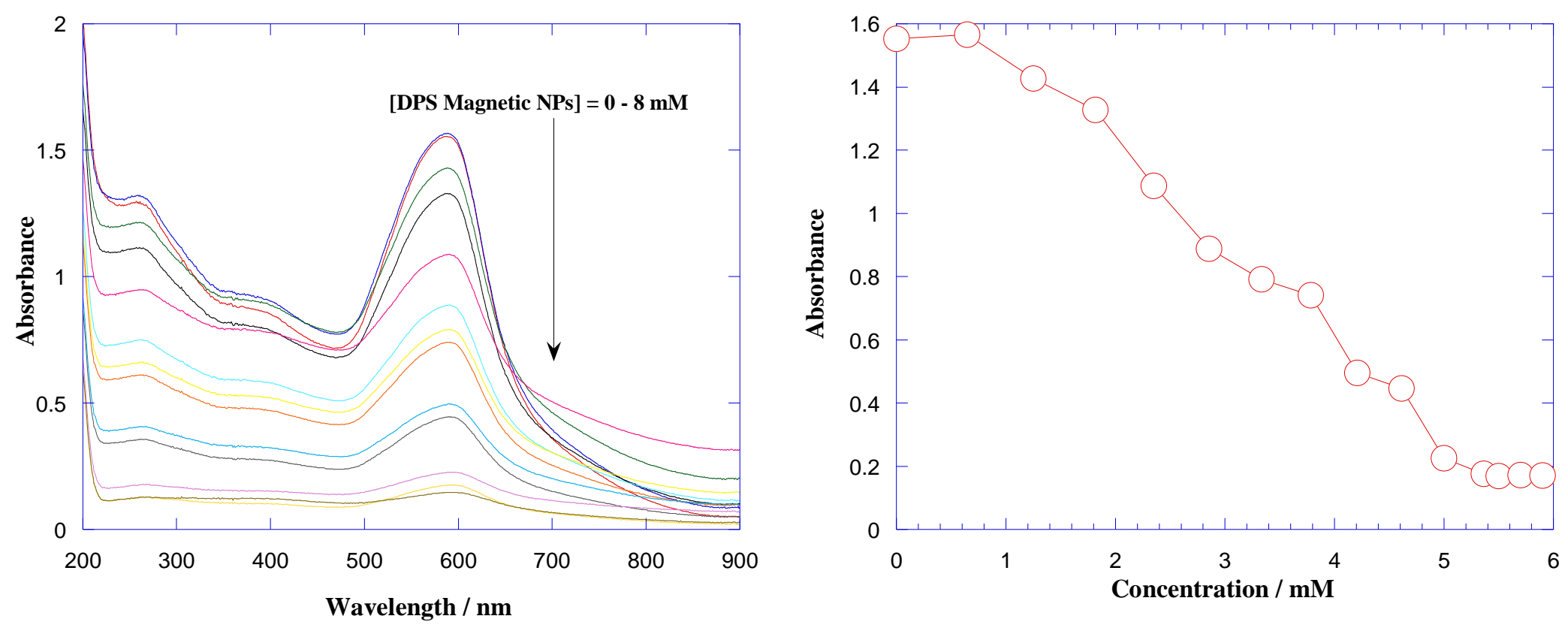

Fig S13. UV-visible scans (left frame) and intensity vs concentration / $\mathrm{mM}$ (right frame) representing the extraction of hemoglobin (in aq CTAB) conjugated Au NPs in the presence of DPS stabilized water soluble magnetic NPs in aqueous bulk. 


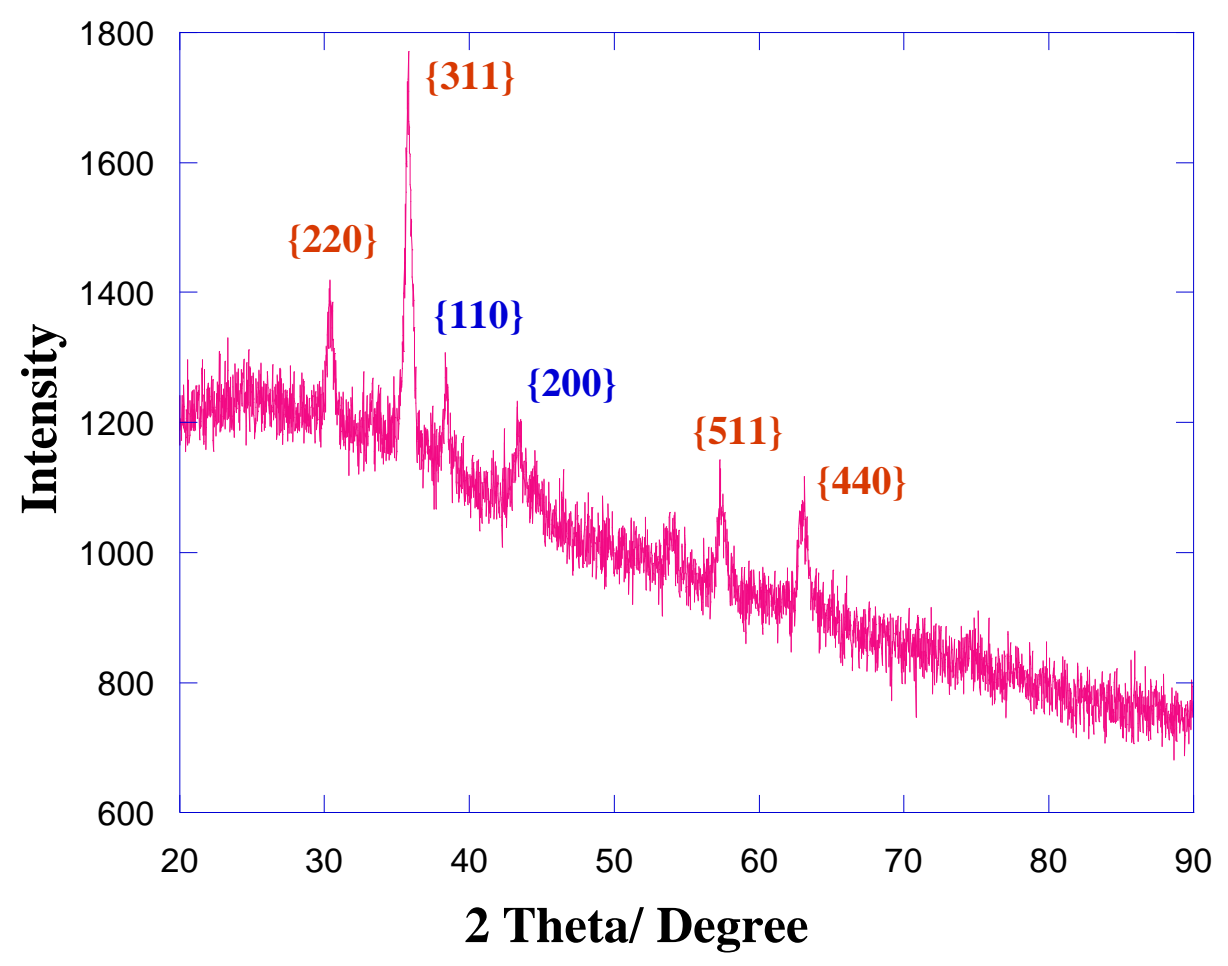

Peaks $\{220\},\{311\},\{511\},\{440\}$ correspond to $\mathrm{Fe}_{3} \mathrm{O}_{4}$ nanoparticles. Peaks $\{110\},\{200\}$ are associated with protein stabilized Au NPs.

Fig S14. XRD patterns of a complexed sample consisting of $\mathrm{Fe}_{3} \mathrm{O}_{4}$ and $\mathrm{Au}$ NPs. 
Fig 15. (a) FESEM image of purified extracted samples of wheat protein (in aqueous (TAB) coated Au NPs by SDS stabilized surface active magnetic NPs (sample \# 6, Table 1) showing the presence of polyhedral NPs. EDS elemental mapping of $\mathrm{Fe}, \mathrm{O}, \mathrm{Au}, \mathrm{C}$, and $\mathrm{N}$ along with EDS spectrum (b). Tabular form of elemental composition of various elements. See details in text.

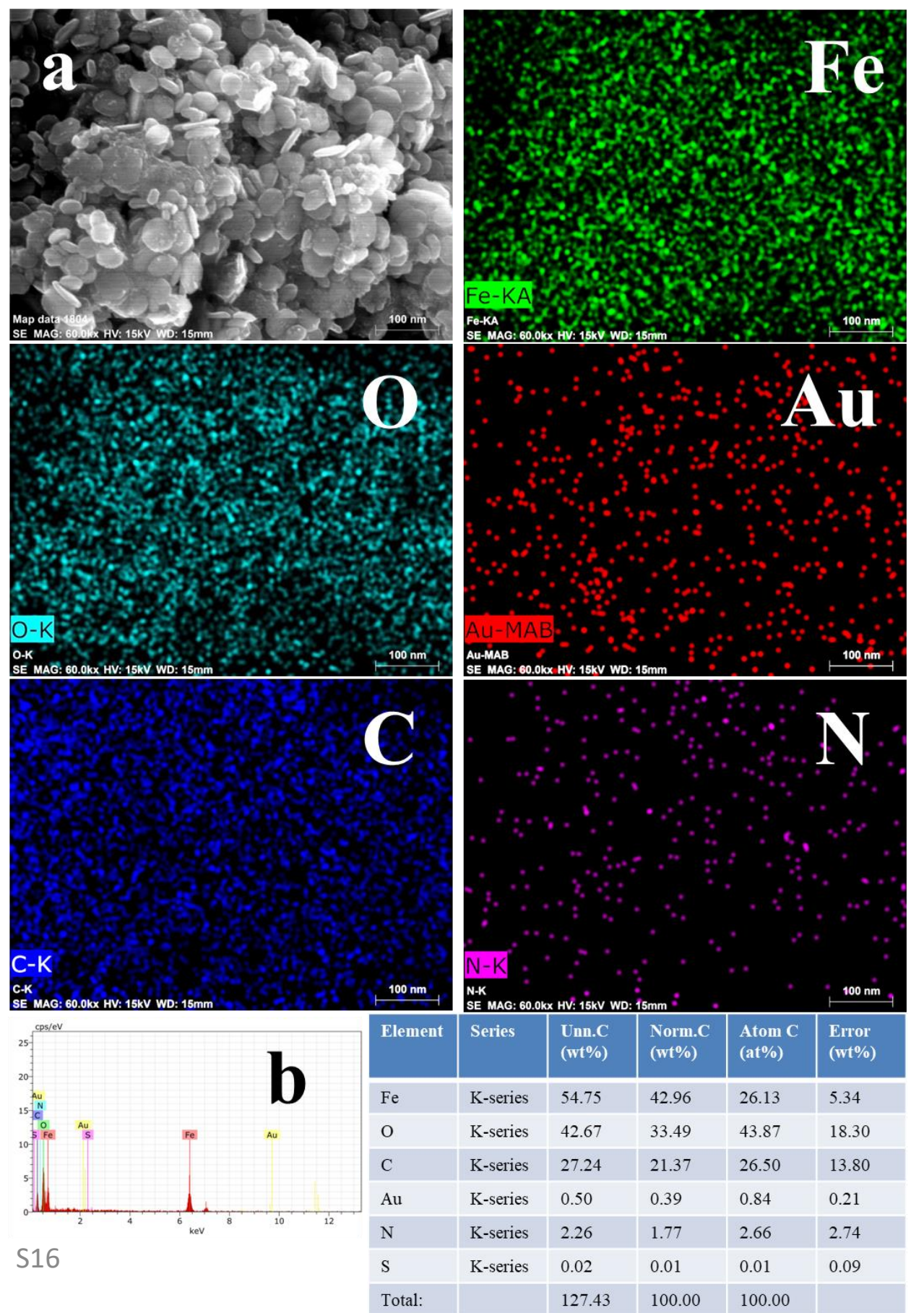

